\title{
Impacto microclimático estival de la configuración de distintas tipologías de espacio abierto en manzanas urbanas. Esquema concentrado versus disperso
}

María-Emilia Balmaceda. Consejo Nacional de Investigaciones Científicas y Técnicas (Conicet), Mendoza, Argentina.

María-Alicia Cantón. Consejo Nacional de Investigaciones Científicas y Técnicas (Conicet), Mendoza, Argentina.

Érica-Norma Correa. Consejo Nacional de Investigaciones Científicas y Técnicas (Conicet), Mendoza, Argentina.

RESUMEN | Tradicionalmente, las manzanas urbanas presentan en su configuración un espacio abierto central como resultado de la sumatoria de patios de viviendas. Dicha estructura ha sufrido modificaciones, dando lugar a un modelo disperso de espacio abierto. Este trabajo evalúa ambos modelos -concentrado, disperso- en manzanas de baja densidad, a fin de determinar el esquema más eficiente desde el punto de vista de su impacto en el microclima urbano. Para esto se caracterizaron escenarios mediante un conjunto de indicadores y se midió la temperatura del aire en los canales viales mediatos a cada manzana. Los datos obtenidos fueron procesados estadísticamente mediante análisis de frecuencia y por componentes principales. Los resultados alcanzados muestran que la distribución dispersa del espacio abierto en verano reduce las temperaturas del entorno, contribuyendo a disminuir el impacto de la trama edilicia sobre el microclima de la ciudad.

PALABRAS CLAVE | planificación urbana, distribución espacial, morfología urbana.

ABSTRACT | Traditionally, the configuration of urban blocks includes a central open space, which results from the addition of lot backyards. However, this configuration has undergone modifications resulting in a spread-out model of open-space. This paper evaluates both models -concentrated and disperse- in low-density blocks, in order to determine the most efficient distribution regarding the impact on urban microclimates. For this purpose, scenarios were characterized by a set of indicators and the air temperature in the urban canyons was measured at each block. Data was processed statistically using frequency analysis and principal components. Results show that the disperse distribution of open space in summer reduces the temperatures of the immediate environment, contributing to reduce the impact of the built tissue on the city's microclimate.

KEYWORDS | urban planning, spatial distribution; urban morphology.

Recibido el 26 de enero de 2017, aprobado el 5 de julio de 2017

E-mails: M.-E. Balmaceda, mbalmaceda@mendoza-conicet.gob.ar | M.-A. Cantón, macanton@mendoza-conicet.gob.ar | E.-N. Correa, ecorrea@mendoza-conicet.gob.ar 


\section{Introducción}

La ciudad de Mendoza, Argentina, es un conglomerado urbano próximo a alcanzar el millón de habitantes, inserto en una zona desértica natural de la región. Desde el punto de vista urbanístico, la estructura del Área Metropolitana, como la del resto de las ciudades hispanoamericanas, está conformada por una retícula espacial en damero con manzanas de formas cuadradas o rectangulares que constituyen el soporte físico de la volumetría edilicia. La estructura del parcelamiento de las mismas está definida por un mosaico de lotes que en su gran mayoría son de 10 metros de frente, profundidad variable y forma rectangular, en donde uno de sus lados menores limita con el espacio urbano de la calle.

La edilicia inserta en dicha trama responde a criterios comunes a los que definieron el proceso de fundación de la ciudad: su estructuración en torno a un espacio abierto; es decir, el patio es el elemento regulador del partido arquitectónico alrededor del cual se distribuyen los espacios interiores relacionados con las actividades de descanso, trabajo y recreación. La sumatoria de dichos patios conforma en el interior de la manzana una unidad mayor: el centro libre de manzana.

Si bien la transformación urbana y edilicia fue un hecho permanente y constante en la segunda mitad del siglo xIx y primera del xx, los cambios operados por la modernidad impactan, entre otros factores, en un mayor uso del suelo regulado por índices que potencian la ocupación de las parcelas y en el surgimiento de nuevas tipologías que abandonan los esquemas abiertos y abren paso a las tipologías compactas.

En este marco, el proceso de renovación y consolidación de las manzanas urbanas ha variado la configuración del espacio abierto, y lo ha disminuido en cantidad. Respecto a su configuración, el modelo de espacio abierto concentrado en el centro de la manzana ha tomado nuevas formas de carácter más disperso. Por otra parte, su cuantificación, a partir del análisis de un conjunto muestral de 37 manzanas en el año 2003 y su comparación con la situación actual, demuestran que en el lapso de una década la pérdida de espacio abierto promedio es del orden del 20\% (Cantón, 2004). Esta pérdida es más significativa en las áreas urbanas residenciales, donde la disminución alcanza valores máximos del 35\%. Tal situación obedece a que, en las modificaciones incorporadas al Código de Edificación del departamento de Capital, Mendoza, a fines de 2010, solo se regularon retiros posteriores en edificaciones que superan los tres pisos, siendo posible que las construcciones de menor altura colinden con el límite posterior del predio.

En consecuencia, el modelo de centro de manzana concentrado en su interior se ha diluido, surgiendo otra tipología asociada a la fragmentación del espacio abierto.

El primer modelo, centro de manzana concentrado, ha sido valorado, defendido culturalmente y regulado a través de las siguientes normativas vigentes en el territorio nacional: plan urbano ambiental de la ciudad de Buenos Aires; ordenanza de ordenamiento territorial y uso del suelo para el partido de La Plata; código de planeamiento urbano de Tucumán; y los códigos de edificación de las ciudades de Córdoba, Bahía Blanca y Rosario. 
El segundo modelo, centro de manzana fragmentado, es el resultado de la incorporación de nuevas tipologías edilicias y de la falta de regulación de los frentes internos de la edilicia.

Estudios previos en torno a otras categorías de espacio abierto, como parques y plazas, llevados a cabo por el Instituto de Ambiente, Hábitat y Energía (INAHE), han demostrado una mayor eficiencia ambiental en torno a la fragmentación y distribución homogénea del espacio vegetado respecto a su concentración (Correa, Martínez, Lesino, de Rosa \& Cantón, 2006) y que dicha eficiencia está asociada a rangos de superficie (Cantón, Fernandez, Mesa $\&$ de Rosa, 2006). En el caso específico de los centros de manzana, se desconoce la eficiencia respecto a la distribución del espacio abierto.

Por otro lado, el Área Metropolitana de Mendoza (Амм) posee una isla de calor urbana (ICU) con un promedio de $6^{\circ} \mathrm{C}$ (invierno-verano), alcanzando máximas de $10^{\circ} \mathrm{C}$. La ICU produce mayores temperaturas del aire en el centro de las ciudades en relación con las áreas rurales circundantes; afecta el consumo de energía residencial, la calidad del aire y el uso de los espacios al aire libre, entre otros aspectos (Pantavou, Theoharatos, Mavrakis \& Santamouris, 2011; Stathopoulou, Mihalakakou, Santamouris \& Bagiorgas, 2008). En invierno, la ICU mitiga la demanda de calefacción; sin embargo, en verano acentúa la demanda de enfriamiento (Correa, de Rosa \& Lesino, 2008). Por tales motivos, el análisis se desarrolló para la temporada estival.

Este trabajo persigue analizar y evaluar ambos modelos de distribución del espacio abierto (concentrado y fragmentado) en el contexto de manzanas urbanas de baja densidad, a los efectos de determinar el esquema más eficiente desde el punto de vista de su impacto en el microclima de los canales viales perimetrales en verano.

El objeto de investigación es el espacio abierto en el interior de las manzanas urbanas. Al respecto, la pregunta de investigación es en qué medida y bajo qué formato -abierto concentrado o abierto disperso- dichos espacios contribuyen de manera más eficiente a mejorar las condiciones térmicas de los canales viales conexos a las manzanas urbanas. Hasta el momento no se ha establecido con precisión la forma más eficiente de distribución del espacio abierto interior en su relación con las condiciones térmicas del lugar. Establecer este parámetro contribuirá a tomar decisiones respecto al ordenamiento de la edilicia en la manzana urbana.

\section{Metodología}

\section{Selección de los casos de estudio}

Con el objeto de identificar diferentes esquemas de distribución de espacios abiertos respecto a la edilicia en manzanas urbanas se seleccionó un universo de análisis de once manzanas correspondientes al área residencial de baja densidad de Capital, Mendoza. A partir de documentación catastral del área, para cada una de esas once manzanas se relevaron sus dimensiones y la superficie cubierta y libre. Los resultados obtenidos permitieron identificar dos esquemas de distribución de espacio abierto, uno asociado a la concentración del mismo en el centro de la manzana y el 
otro a una distribución fragmentada, ya sea en forma regular o irregular. De acuerdo con el objetivo del trabajo, se seleccionaron dos casos de estudio:

- Caso 1: El espacio abierto se concentra en el centro de la manzana.

- Caso 2: El espacio abierto bordea el centro de la manzana y se distribuye en forma homogénea.

En ambos casos la superficie de la manzana es semejante, al igual que la relación porcentual entre espacio abierto y construido:

- Caso 1: $11.100 \mathrm{~m}^{2}, 52 \%$ superficie construida y $48 \%$ superficie abierta.

- Caso 2: $12.460 \mathrm{~m}^{2}, 56 \%$ superficie construida y $44 \%$ superficie abierta.

Los casos se caracterizan por ser contrastantes entre sí en la distribución del espacio abierto; son representativos del entorno y pertenecen a la misma área de influencia. A modo de referencia, algunos autores abordan similar metodología en el diseño experimental (Ruiz \& Correa, 2015a; 2015b).

En la figura 1 se puede observar la localización del área de estudio y las manzanas relevadas y los casos seleccionados. FIgURA I | Área Metropolitana de
Mendoza, Argentina

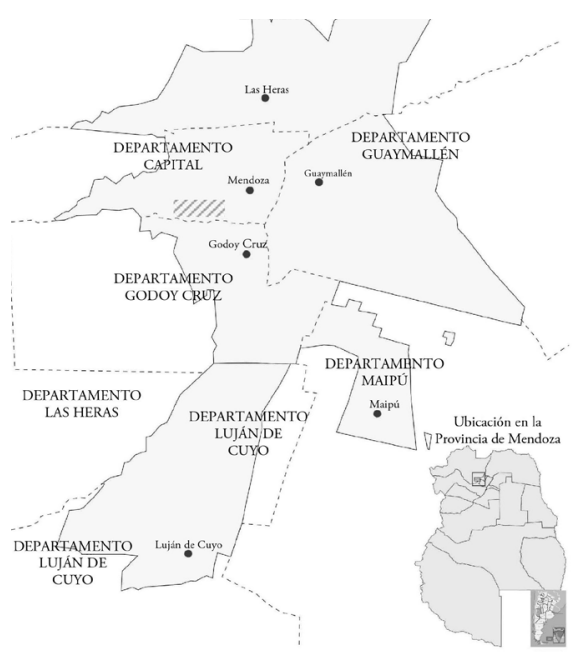

Manzanas seleccionadas del área de estudio-baja densidad- pertenecientes y sector de estudio al distrito "Quinta Sección", Mendoza Capital

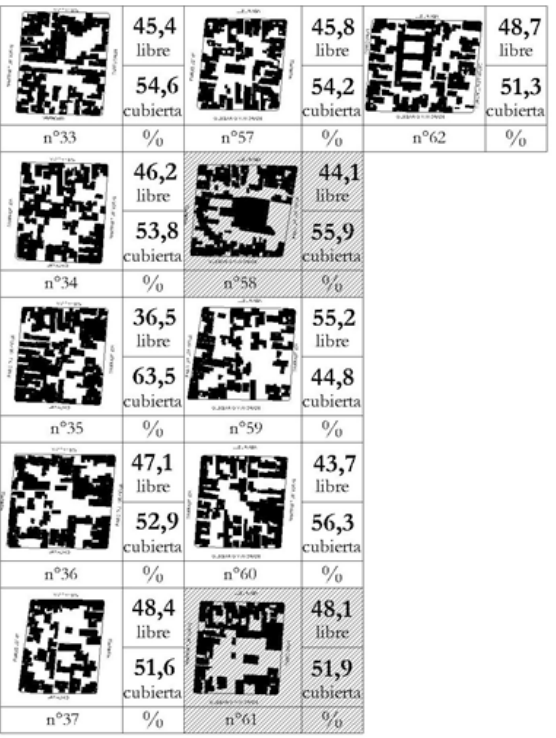

NOTA SE RESALTAN EN GRIS LOS DOS CASOS A ANALIZAR (CASO I ABAJO Y CASO 2 ARRIBA)

FUENTE ELABORACIÓN PROPIA 


\section{Caracterización de los casos de estudio}

Para describir los casos de estudio se seleccionó un conjunto de variables e indicadores, agrupados en torno a distintos aspectos:

- Emplazamiento del canal vial y su acceso al sol: azimut superficial, horas de sol, relación azimut superficial/horas de sol, azimut solar en temperatura máxima.

- Estructura morfológica del canal vial: factor de visión de cielo (svf).

- Estructura morfológica de la manzana: volumen edificado, compacidad v/s, altura media edificada, relación superficie sellada/superficie abierta, relación superficie abierta central/superficie abierta total, factor de ocupación del suelo (FOS) y factor de ocupación total (FОT).

- Propiedades ópticas de los materiales: albedo combinado (resulta del promedio del albedo de los materiales, ponderado por área horizontal de la manzana) y emisividad combinada (resulta del promedio de la emisividad de los materiales, ponderada por área horizontal de la manzana).

Tal caracterización tiene por objeto definir las particularidades de cada manzana en estudio y establecer relaciones entre los indicadores y las temperaturas microclimáticas puntuales obtenidas en los canales viales que flanquean los límites de estas manzanas urbanas.

\section{Determinación de indicadores}

\section{Factor de visión de cielo (SVF)}

El factor de visión de cielo (svf: sky view factor) es un parámetro utilizado para caracterizar las condiciones radiativas para un punto de observación, que expresa la relación entre el área visible de cielo y la porción de bóveda celeste cubierta por los distintos componentes del medio.

Para el cálculo del factor de visión de cielo se tomaron imágenes hemisféricas mediante cámara digital Nikon CoolPix con lente ojo de pez. Las mismas se procesaron mediante el software Pixel de Cielo desarrollado por la Unidad de Investigación y Desarrollo (Correa, Pattini, Córica, Fornés \& Lesino, 2006). Los valores obtenidos son resultados de cinco pruebas realizadas por imagen, ajustando los rangos de delineación de colores presentes en el cielo, variando el valor de SVF, hasta llegar a la imagen que refleja con mayor precisión las condiciones reales de la imagen original.

\section{Recorrido solar}

El recorrido solar fue obtenido mediante el programa GEOsOL v.2.o. Es una herramienta computacional para el cálculo de coordenadas solares y la estimación de irradiación solar horaria (Hernández, 2003). Permite obtener, para cualquier lugar y día del año, los siguientes valores numéricos: hora solar de salida y puesta del sol sobre el horizonte; duración del día; diferencia horaria entre hora solar y hora oficial; declinación solar para la fecha consignada; ángulo horario, altitud y azimut solares, hora por hora, desde la salida hasta la puesta del sol; e irradiación solar directa, difusa y total en $\mathrm{mJ} / \mathrm{m}^{2}$, hora por hora, sobre cualquier superficie especificada por el usuario (Duffie \& Beckman, 1991). 
El programa antedicho genera un conjunto de gráficos con los cuales se representa: la evolución horaria de las irradiaciones solares directa, difusa y total mediante un gráfico de barras; la trayectoria del sol sobre la bóveda celeste en 2D (carta solar) y 3D (perspectiva isométrica); y gráfica de obstáculos a la trayectoria del sol en 2D. En este caso, para el cálculo del recorrido solar se utilizó el método de día claro de Hottel, el cual utiliza la ecuación de Hottel.

Los indicadores restantes fueron determinados de acuerdo con su definición:

- Volumen: cubica el volumen edificado del canal.

- Compacidad (volumen/superficie): relaciona el volumen edificado con la superficie total del cuadrante.

- Altura media edilicia: promedio de las alturas de las fachadas que se encuentran en cada cuadrante.

- Relación sellado/abierto: indica la proporción de espacio sellado respecto al espacio abierto y su distribución espacial en la manzana.

- Relación abierto central/abierto total: indica la proporción de espacio abierto y su distribución espacial en la manzana.

- Factor de ocupación del suelo (FOs): valor que indica el porcentaje del área de terreno construida en una zona determinada.

- Factor de ocupación total (ғОт): relación del total de la superficie edificada y superficie total de la parcela.

- Albedo combinado: resulta del promedio del albedo de los materiales, ponderado por área horizontal en la manzana.

- Emisividad combinada: resulta del promedio de la emisividad de los materiales, ponderada por área horizontal en la manzana.

Estos indicadores se calcularon a partir de planos catastrales, relevamiento en campo y a partir de imágenes satelitales. Los valores de emisividad y albedo fueron tomados de estudios previos (Alchapar, Correa \& Cantón, 2014).

\section{Monitoreo de los casos de estudio}

El método utilizado consistió en medir de forma continua la temperatura del aire en los canales viales mediatos a cada manzana en estudio mediante dataloggers del tipо ново. El instrumental de medición fue colocado dentro de una caja de material plástico perforada en cuatro de sus caras, con el objeto de evitar la irradiación del sensor y asegurar una adecuada circulación de aire. Las mediciones se desarrollaron en la estación de verano en 2015 y 2016, durante 58 días, divididos en dos periodos: el primero desde el 22 de enero al 26 de febrero de 2015, y el segundo del 14 de diciembre de 2015 al 4 de enero de 2016. Los datos se registraron cada 15 minutos en la siguiente configuración: un datalogger por canal vial, que bordea los límites de la manzana, posicionado a una altura promedio de 2 metros desde el nivel de la calle (Oke, 2006). 
Selección de los días de estudio y justificación de los días seleccionados como representativos

Los datos obtenidos de las mediciones fueron promediados en forma horaria para una mejor comprensión de los comportamientos globales. A partir de ello se seleccionaron, para los dos períodos medidos, días con condiciones estables y representativas de un día típico de verano en Mendoza, definido a partir de los siguientes parámetros: heliofanía relativa superior al 70\%, días despejados, donde la nubosidad es menor o igual a 3/8, con velocidades de viento inferiores a $6 \mathrm{~km} / \mathrm{h}$; humedad relativa media, inferior al 50\%. Se identificó un total de 30 días (21 días correspondientes a febrero de 2015 y 9 días a diciembre de 2015 y enero de 2016).

Para cada uno de los 58 días monitoreados se obtuvo la temperatura máxima, media y mínima como un descriptor del comportamiento. Posteriormente se seleccionaron 30 días, con 30 valores de temperatura máxima, 30 valores de temperaturas medias y 30 valores de temperaturas mínimas, para cada localización comparada (norte, sur, este y oeste), para las dos manzanas en estudio; es decir, se compararon 240 valores de temperaturas máximas, 240 de medias y 240 de mínimas.

La primera comparación se realizó con los datos resultantes de las mediciones promediadas hora a hora, de acuerdo con el análisis comparativo del total de los días monitoreados (58) respecto a los días seleccionados (30). Se observó que el mayor impacto está en la mínima, debido a que en los días seleccionados se excluyeron los días fríos. Este análisis mejora el comportamiento de los datos evaluados, para que los mismos sean comparativos entre sí (tabla 1).

Para mejorar la desviación estándar y la varianza de las variables indicadoras hora a hora, se tomaron tres indicadores (máxima, mínima y media), utilizados de manera frecuente para describir el comportamiento de la curva de temperatura del aire de un lugar. En este análisis se observó que las diferencias más significativas se producen en los patrones de enfriamiento; en los patrones de calentamiento no se observa significativa varianza ni desviación estándar (tabla 1). 


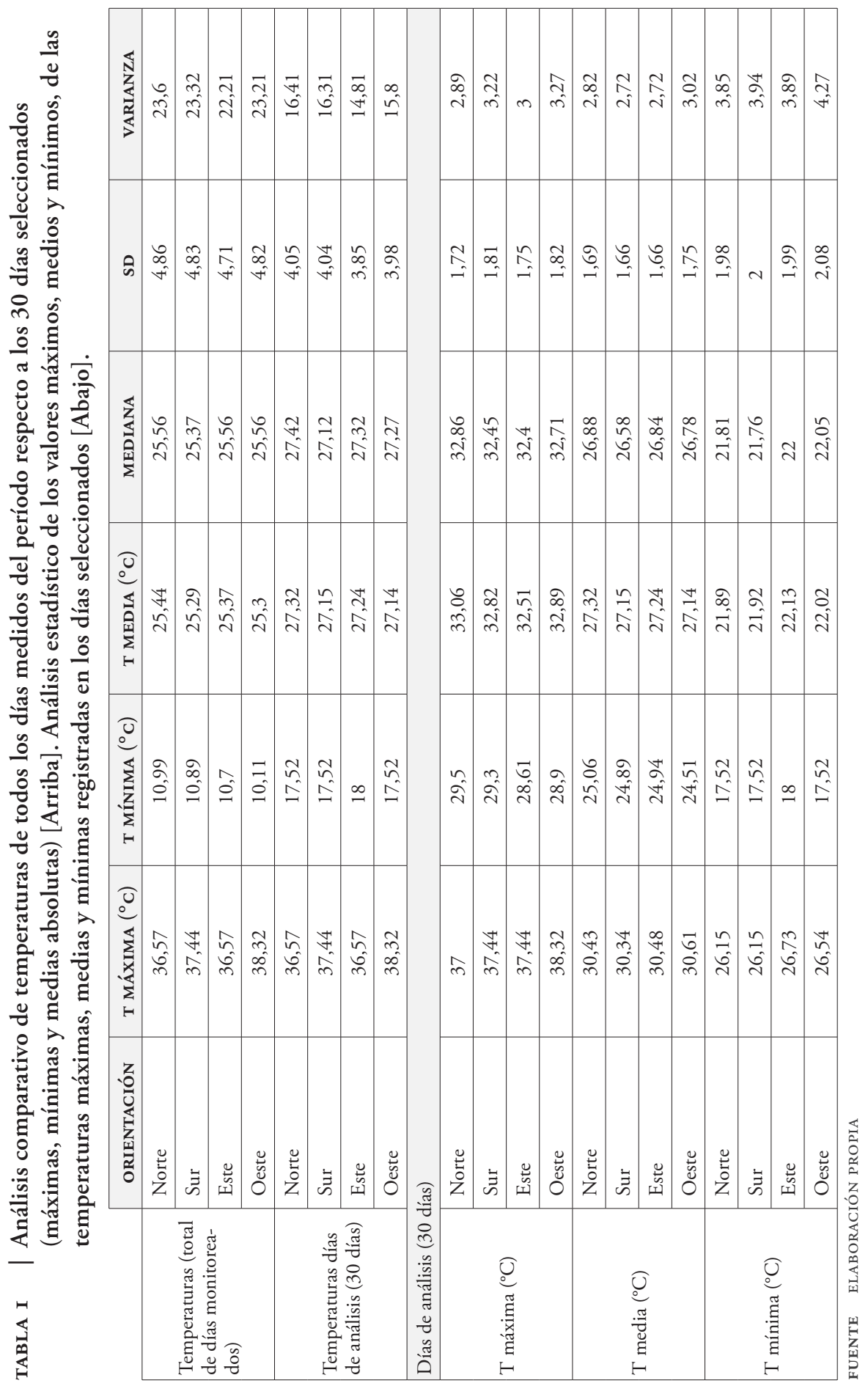




\section{Resultados}

A continuación se presenta la distribución de frecuencia del total de los días monitoreados, de los 30 días seleccionados medidos hora a hora y de los indicadores de referencia. Los datos obtenidos fueron agrupados y procesados estadísticamente de dos maneras: primero mediante un análisis de frecuencia y luego mediante un análisis por componentes principales (ACP).

\section{Análisis de frecuencia}

Los valores de la temperatura del aire, obtenidos en forma horaria en cada uno de los puntos de monitoreo, han sido analizados en forma comparativa para los dos casos de estudio a partir del análisis de las diferencias de temperaturas horaria, entre la manzana con el espacio abierto concentrado (caso 1) y la manzana con el espacio abierto disperso (caso 2). Este análisis se realizó para el total de los días monitoreados ( 58 días) y para un conjunto de 30 días seleccionados por ser representativos de las condiciones típicas de verano de la ciudad de Mendoza, como se discutió anteriormente (véase tabla 1). También se tomaron tres indicadores $-\mathrm{T}$ máxima, T media y T mínima- para el conjunto de 30 días representativos y se evaluó la diferencia de temperatura entre ambos casos para cada orientación monitoreada (tabla 2).

TABLA 2 Porcentaje de valores (+) y (-) como resultado de la diferencia de las temperaturas de la manzana con espacio abierto concentrado (caso 1 ) respecto a la dispersa (caso 2)

\begin{tabular}{|l|l|c|c|}
\hline \multicolumn{1}{|c|}{ DÍAs } & ORIENTACión & $\Delta \mathbf{T ~ C ~ - ~ D ~ ( + ) ~ ( \% ) ~}$ & $\Delta \mathbf{T ~ C ~ - ~ D ~ ( - ) ~ ( \% ) ~}$ \\
\hline \multirow{5}{*}{ Todos los días medidos (58) } & Norte & 70,69 & 29,31 \\
\cline { 2 - 4 } & Sur & 53,95 & 46,05 \\
\cline { 2 - 4 } & Este & 65,45 & 34,55 \\
\cline { 2 - 4 } & Oeste & 81,32 & 18,68 \\
\cline { 2 - 4 } & TOTAL & 67,85 & 32,15 \\
\hline \multirow{4}{*}{30 días seleccionados } & Norte & 71,53 & 28,47 \\
\cline { 2 - 4 } & Sur & 52,92 & 47,08 \\
\cline { 2 - 4 } & Este & 67,5 & 32,5 \\
\cline { 2 - 4 } & Oeste & 82,92 & 17,08 \\
\cline { 2 - 4 } & TOTAL & 68,72 & 31,28 \\
\hline
\end{tabular}

NOTA HORA A HORA, EN EL TOTAL DE DÍAS DE MEDICIÓN Y EN LOS 30 DÍAS SELECCIONADOS.

FUENTE ELABORACIÓN PROPIA 


\section{Cálculo de frecuencias hora a hora por orientación}

En términos generales, los resultados obtenidos muestran mayores temperaturas en el caso de la manzana con condiciones del espacio abierto concentrado (caso 1) en los cuatro canales viales (orientaciones) que bordean a la misma. En ambos análisis horarios (58 y 30 días) se observó que alrededor del 68\% de las condiciones monitoreadas mostraba esta tendencia, la cual se expresa con mayor énfasis en los casos con orientación oeste $(82 \%)$ y norte $(71 \%)$, y es menos notoria en los casos monitoreados con orientación sur (53\%) (tabla 2).

\section{Cálculo de frecuencias de temperaturas (máximas, medias y mínimas) por orientación}

En términos generales, se mantiene la tendencia del análisis por frecuencia horaria, donde el entorno de una manzana con el espacio abierto concentrado (caso 1) muestra mayores temperaturas que el entorno de una manzana con espacio abierto disperso (caso 2). Tanto en las máximas como en las medias y las mínimas se muestra mayor frecuencia de rangos positivos como resultado de la diferencia de temperaturas del caso 1 respecto al caso 2 .

Respecto de las máximas, la manzana con el espacio abierto concentrado (caso 1) presenta temperaturas superiores a la manzana con espacio abierto disperso (caso 2). En las cuatro orientaciones, esta tendencia de las temperaturas máximas se da en un $71 \%$ de los casos; en el caso de las mínimas, en un 78\%; y en cuanto a las medias, la tendencia es menos evidente, con un 58\%. Es decir, la tendencia se observó más claramente en las máximas y en las mínimas. En el caso de las temperaturas medias no fue tan significativa (tabla 3 ).

Al igual que en el análisis de frecuencia horaria, se observa que en el sur es donde se aprecian las menores diferencias, del orden del 60\%. Las orientaciones que muestran mayor recurrencia de temperaturas de manzanas con espacio abierto concentrado (caso 1) son este y oeste, con alrededor del $80 \%$, a diferencia del análisis horario, donde las orientaciones con mayor temperatura en la manzana que presenta el espacio abierto concentrado eran norte y oeste (tabla 3 ).

En las máximas, la orientación que tiene mayor incidencia es la oeste, con rangos del orden del 87\%; y la de menor incidencia es la sur, con un 61\% (tabla 3).

Las medias muestran la misma tendencia, pero con menor diferenciación, ya que la tendencia global es del orden del 60\%. La orientación que más destaca es la oeste, con un $84 \%$, y las de menor incidencia son la norte y la sur, con un $60 \%$ (tabla 3 ).

En las mínimas, la orientación que más diferenciación mostró fue la oeste, con un valor de $82 \%$, mientras la de menor incidencia fue la sur, con un 55\% (tabla 3 ). 
TABLA 3 | Porcentaje de valores (+) y (-) como resultado de la diferencia las temperaturas de la manzana con espacio abierto concentrado (caso 1) respecto a la dispersa (caso 2). Máximas, medias y mínimas

\begin{tabular}{|c|c|c|c|}
\hline TEMPERATURA & ORIENTACIÓN & $\Delta \mathrm{T} \mathrm{C}-\mathrm{D}(+)(\%)$ & $\Delta \mathrm{T} \mathrm{C}-\mathrm{D}(-)(\%)$ \\
\hline \multirow{5}{*}{ T máxima } & Norte & 65,22 & 34,78 \\
\hline & Sur & 60,87 & 39,13 \\
\hline & Este & 82,61 & 17,39 \\
\hline & Oeste & 86,96 & 13,04 \\
\hline & TOTAL & 70,83 & 29,17 \\
\hline \multirow{5}{*}{$\mathrm{T}$ media } & Norte & 60 & 40 \\
\hline & Sur & 60 & 40 \\
\hline & Este & 80 & 20 \\
\hline & Oeste & 84,21 & 15,79 \\
\hline & TOTAL & 58,33 & 41,67 \\
\hline \multirow{5}{*}{ T mínima } & Norte & 61,11 & 38,89 \\
\hline & Sur & 55,56 & 44,44 \\
\hline & Este & 77,78 & 22,22 \\
\hline & Oeste & 82,35 & 17,65 \\
\hline & TOTAL & 78,33 & 21,67 \\
\hline
\end{tabular}

FUENTE ELABORACIÓN PROPIA

De acuerdo con la diferencia de temperaturas máximas, medias y mínimas, en los días monitoreados se verificó mayor porcentaje de rangos de frecuencia positivos para las cuatro orientaciones de los canales viales, observándose que la tendencia general indica que el entorno de la manzana con distribución concentrada del espacio abierto (caso 1) se encuentra más caliente que la manzana con espacio abierto disperso (caso 2). Las diferencias promedio entre ambos casos fue, en las temperaturas máximas, del orden de $0,61^{\circ} \mathrm{C}$, en el $71 \%$ de los días evaluados; en las temperaturas medias, del orden de $0,23^{\circ} \mathrm{C}$, en el $59 \%$ de los días evaluados; y en las mínimas, de $0,41{ }^{\circ} \mathrm{C}$, en el $78 \%$ de los días evaluados (tabla 4 ).

TABLA 4 Diferencia del promedio de temperaturas máximas, medias y mínimas, cuantificadas en grados centígrados, de los dos casos planteados

\begin{tabular}{|c|l|c|c|c|c|}
\hline \multirow{2}{*}{$\begin{array}{c}\text { TEMPE- } \\
\text { RATURAS }\end{array}$} & $\begin{array}{c}\text { ORIEN- } \\
\text { TACIÓN }\end{array}$ & $\begin{array}{c}\text { CASO I - MZ. } \\
\text { CONCENTRADA } \\
\text { (T PROMEDIO) }\end{array}$ & $\begin{array}{c}\text { CASO 2 - MZ. } \\
\text { DISPERSA } \\
\text { (T PROMEDIO) }\end{array}$ & $\Delta$ T (C-D) & $\begin{array}{c}\text { \% DE DÍAS EN QUE LA } \\
\text { MZ. CONCENTRADA } \\
\text { RESULTA MÁS CALIENTE } \\
\text { QUE LA DISPERSA }\end{array}$ \\
\hline \multirow{2}{*}{ T máxima $\left({ }^{\circ} \mathrm{C}\right)$ ) } & Norte & 33,41 & 32,66 & 0,75 & 97 \\
\cline { 2 - 6 } & Sur & 33,56 & 32,88 & 0,68 & 73 \\
\cline { 2 - 6 } & Este & 33,78 & 33,29 & 0,48 & 50 \\
\cline { 2 - 6 } & Oeste & 32,74 & 32,20 & 0,54 & 63 \\
\hline \multicolumn{2}{|l}{ Promedio }
\end{tabular}


(continuación)

\begin{tabular}{|c|c|c|c|c|c|}
\hline $\begin{array}{l}\text { TEMPE- } \\
\text { RATURAS }\end{array}$ & $\begin{array}{l}\text { ORIEN- } \\
\text { TACIÓN }\end{array}$ & $\begin{array}{l}\text { CASO I - MZ. } \\
\text { CONCENTRADA } \\
\text { (T PROMEDIO) }\end{array}$ & $\begin{array}{c}\text { CASO } 2 \text { - MZ. } \\
\text { DISPERSA } \\
\text { (T PROMEDIO) }\end{array}$ & $\Delta \mathrm{T}(\mathrm{C}-\mathrm{D})$ & $\begin{array}{c}\text { \% DE DÍAS EN QUE LA } \\
\text { MZ. CONCENTRADA } \\
\text { RESULTA MÁS CALIENTE } \\
\text { QUE LA DISPERSA }\end{array}$ \\
\hline \multirow{4}{*}{$\mathrm{T}$ media $\left({ }^{\circ} \mathrm{C}\right)$} & Norte & 27,34 & 27,17 & 0,16 & 80 \\
\hline & Sur & 26,04 & 25,94 & 0,09 & 27 \\
\hline & Este & 27,61 & 27,42 & 0,18 & 53 \\
\hline & Oeste & 27,07 & 26,59 & 0,48 & 77 \\
\hline \multicolumn{4}{|l|}{ Promedio } & 0,23 & 59 \\
\hline \multirow{4}{*}{ T mínima $\left({ }^{\circ} \mathrm{C}\right)$} & Norte & 21,37 & 21,07 & 0,30 & 63 \\
\hline & Sur & 21,86 & 21,65 & 0,20 & 70 \\
\hline & Este & 22,30 & 21,98 & 0,31 & 87 \\
\hline & Oeste & 22,31 & 21,50 & 0,81 & 93 \\
\hline \multicolumn{4}{|l|}{ Promedio } & 0,41 & 78 \\
\hline
\end{tabular}

FUENTE ELABORACIÓN PROPIA

\section{Análisis por componentes principales (ACP)}

De acuerdo con el objetivo de la investigación, se observa que es apresurado afirmar que el comportamiento térmico del entorno de una manzana con el espacio abierto concentrado es más caliente que el entorno de una manzana con espacio abierto disperso, tomando como prueba solo el análisis de frecuencia de temperaturas. Para corroborar si el comportamiento observado está relacionado con las características de la manzana, se decidió aplicar un análisis multivariado. Se realizó un ACP para valorar estadísticamente qué características de la manzana son responsables en mayor grado del comportamiento térmico de sus alrededores.

Para realizar este análisis se determinó un conjunto de indicadores descriptivos de los dos casos de estudio (véase Metodología, segundo inciso: Caracterización de los casos de estudio). Cada una de las manzanas fue dividida en cuatro cuadrantes, correspondientes a las cuatro orientaciones (norte, sur, este y oeste), a través de diagonales.

A continuación, en la tabla 5, a modo de ejemplo se hallan cuantificados los indicadores utilizados en ambas manzanas (casos 1 y 2 ) y para sus cuatro cuadrantes en un día representativo (13 de febrero de 2015). 


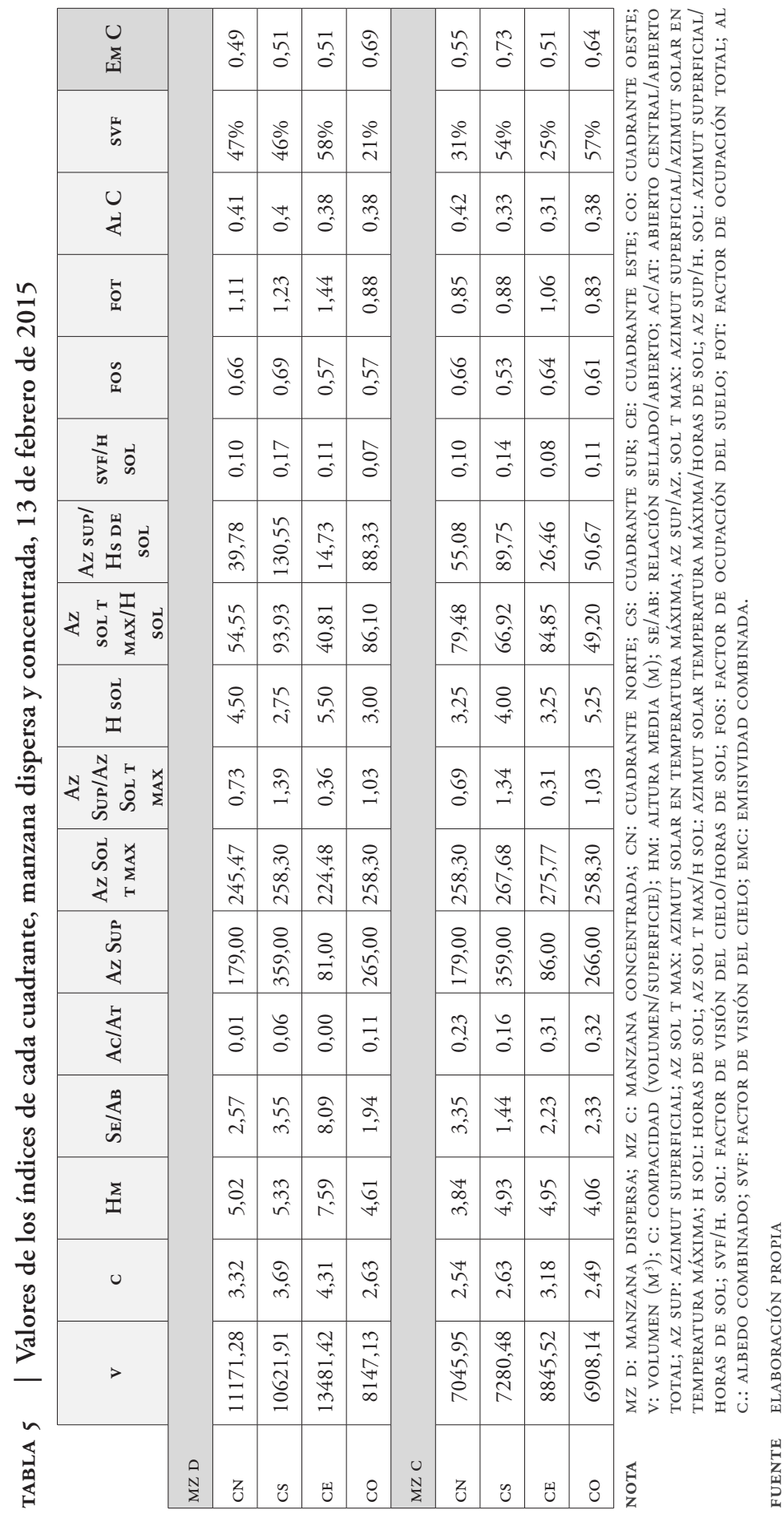




\section{Selección de indicadores para el desarrollo del análisis por componentes principales (ACP)}

Con los indicadores elegidos se construyó una matriz de correlaciones para los dos días representativos seleccionados. Se eligieron aquellos indicadores cuyo coeficiente de correlación de Pearson fue superior a 0,5 y que presentan además baja colinealidad. A modo de ejemplo, se presenta en la tabla 6 la matriz de correlaciones para las temperaturas máximas. El mismo procedimiento se realizó para las temperaturas medias y mínimas.

El conjunto de indicadores que forma parte del análisis final es representativo de la morfología, el recorrido solar en relación con el emplazamiento y la tecnología de los materiales que conforman la manzana urbana. $\mathrm{Y}$ en conjunto explican entre el $86 \%$ y el $99 \%$ de las temperaturas máximas, medias y mínimas de las manzanas evaluadas para el $71 \%$ a $100 \%$ de los casos en el período de análisis.

Se presenta en forma discriminada el análisis por componentes principales para las temperaturas máximas, medias y mínimas.

\section{Temperaturas máximas}

Para el caso de las temperaturas máximas y de acuerdo con análisis de la matriz de correlaciones, fueron seleccionados los siguientes indicadores:

- Relación abierto centrallabierto total: indica la proporción de espacio abierto y su distribución espacial en la manzana.

- Azimut superficial/horas de sol: de acuerdo con la orientación de la fachada, el aumento en la cantidad de horas de sol produce un aumento en las temperaturas. Utiliza la cantidad de horas de sol, relacionada con el recorrido solar y su relación con la orientación como elemento predictivo.

- Albedo combinado: resulta del promedio del albedo o reflectancia de los materiales, ponderado por área horizontal en la manzana. 


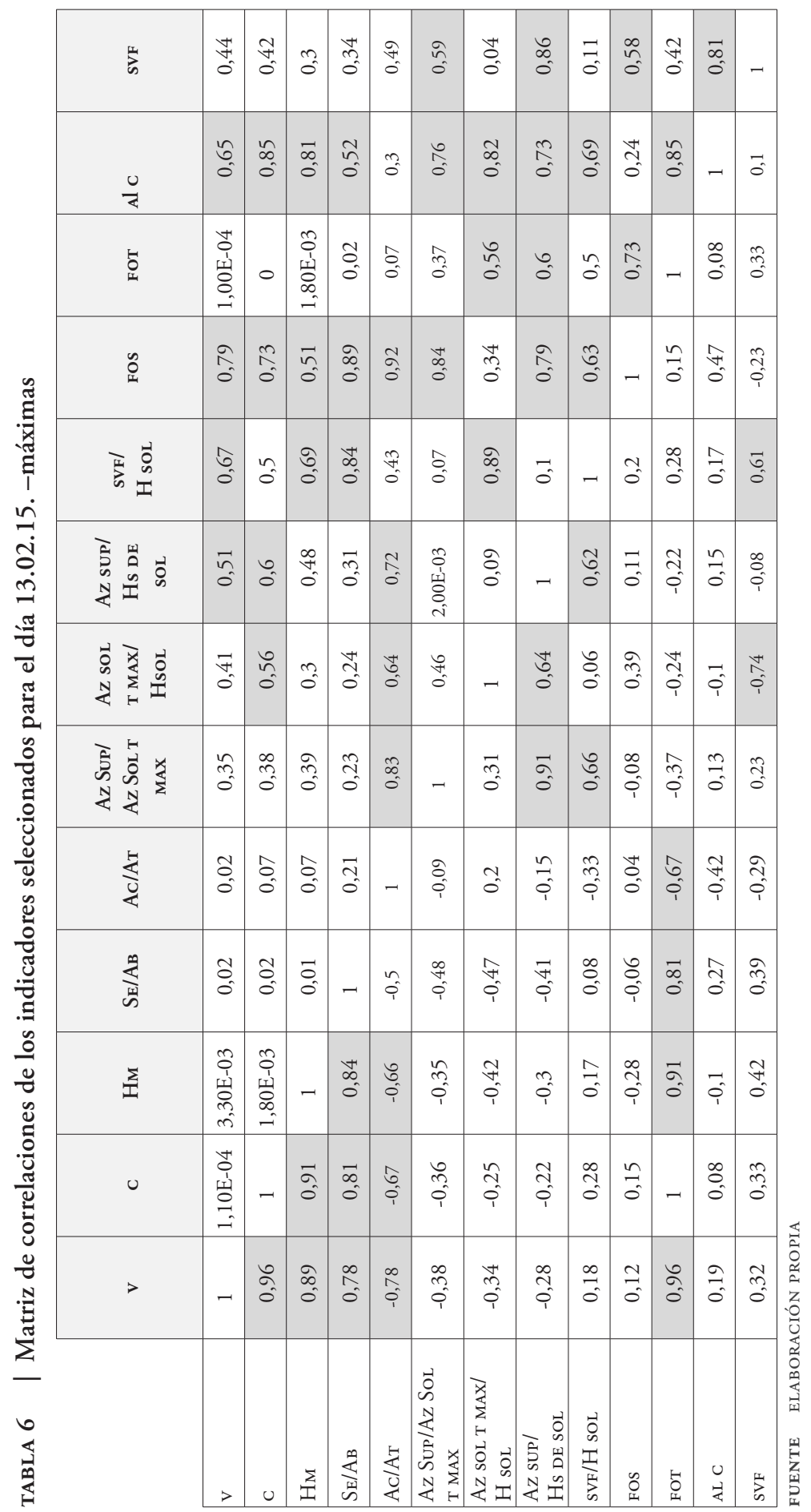


Se realizó un ACP con el objeto de establecer si la forma de distribución de las superficies abiertas en la manzana tiene un impacto significativo sobre el desempeño térmico de la misma.

Los resultados alcanzados muestran que, de 30 días analizados, el comportamiento térmico de 26 días se explica entre un $86 \%$ y $99 \%$ por el efecto de tres indicadores: relación abierto central/abierto total, azimut superficial/horas de sol, y albedo combinado (tabla 7).

La variable objeto de estudio -relación abierto central/abierto total- explica el fenómeno, en primer lugar, en un 11,54\% de las veces con rangos de explicación del $43 \%$ al 47\%; en segundo lugar, en un $23,08 \%$ de las veces con rangos del $27 \%$ al 33\% de explicación; y en tercer lugar, en el 65,38\% de las veces con rangos del $15 \%$ al $21 \%$.

TABLA 7 Resultados de ACP con relación abierto central/abierto total explicada en segundo lugar para el día 16.02.15 (izq.) y en tercer lugar para el día 25.01.15 (der.)

\begin{tabular}{|c|c|c|c|c|c|c|}
\hline \multicolumn{7}{|c|}{ AUTOVALORES } \\
\hline LAMBDA & VALOR & PROPORCIÓN & $\begin{array}{l}\text { PROP. } \\
\text { ACUM. }\end{array}$ & VALOR & PROPORCIÓN & $\begin{array}{l}\text { PROP. } \\
\text { ACUM. }\end{array}$ \\
\hline 1 & 1,74 & 0,44 & 0,44 & 2,1 & 0,52 & 0,52 \\
\hline 2 & 1,34 & 0,33 & 0,77 & 1,07 & 0,27 & 0,79 \\
\hline 3 & 0,79 & 0,2 & 0,97 & 0,79 & 0,2 & 0,99 \\
\hline 4 & 0,13 & 0,03 & 1 & 0,04 & 0,01 & 1 \\
\hline \multicolumn{7}{|c|}{ Correlaciones con las variables originales } \\
\hline Variables & $\mathrm{CP} 1$ & CP 2 & CP 3 & $\mathrm{CP} 1$ & $\mathrm{CP} 2$ & CP 3 \\
\hline T máx. $\left({ }^{\circ} \mathrm{C}\right)$ & 0,79 & 0,54 & $-0,2$ & 0,9 & $-0,36$ & 0,22 \\
\hline AclAt & $-0,24$ & 0,94 & $-0,18$ & $-0,55$ & $-0,43$ & 0,72 \\
\hline Az Sup/H. Sol & 0,7 & 0,13 & 0,7 & 0,19 & 0,87 & 0,46 \\
\hline $\mathrm{Al} \mathrm{C}$ & 0,76 & $-0,38$ & $-0,49$ & 0,98 & $-0,08$ & 0,1 \\
\hline
\end{tabular}

FUENTE ELABORACIÓN PROPIA

Para el total de los días evaluados, el análisis por componentes principales ACP de las temperaturas máximas indica que en un $73 \%$ de los días la manzana con una distribución dispersa del espacio abierto (caso 2), es más fresca respecto de la manzana con el espacio abierto concentrado en el centro (caso 1). Es decir, en la tabla de correlaciones con las variables originales (tabla 7), cuando la relación espacio abierto central respecto al espacio abierto total crece, la temperatura máxima aumenta.

\section{Temperaturas medias}

Para el caso de las temperaturas medias y de acuerdo con el análisis de la matriz de correlaciones, fueron seleccionados los mismos indicadores que en las máximas: relación abierto central/abierto total, azimut superficial/horas de sol y albedo combinado. 
Los resultados del ACP mostraron que, de 30 días analizados, el comportamiento térmico de 24 días se explica entre un $88 \%$ y $98 \%$ debido al efecto de estos tres indicadores (véase como ejemplo tabla 8).

La variable objeto de estudio -relación abierto central/abierto total- explica las temperaturas medias de la manzana en primer lugar en un $50 \%$ de las veces, con rangos de explicación entre el 39\% al 54\%; en segundo lugar, un 8,33\% de las veces con rangos de $30 \%$ a $32 \%$; y en tercer lugar, el $41,66 \%$ de las veces con rangos de $15 \%$ a $20 \%$.

Para el total de los días evaluados, el análisis por componentes principales ACP de las temperaturas medias indica que en el 100\% de los días la manzana con una distribución dispersa del espacio abierto (caso 2), es más fresca respecto de la manzana con el espacio abierto concentrado (caso 1). Es decir, en la tabla de correlaciones con las variables originales (tabla 8), cuando la relación espacio abierto central respecto al espacio abierto total crece, la temperatura media aumenta.

TABLA 8 Resultados de ACP con relación abierto central/abierto total explicada en primer lugar para el día 11.02.15 (izq.) y explicada en segundo lugar para el día 22.01.15 (der.)

\begin{tabular}{|c|c|c|c|c|c|c|}
\hline \multicolumn{7}{|c|}{ AUTOVALORES } \\
\hline LAMBDA & VALOR & PROPORCIÓN & $\begin{array}{l}\text { PROP. } \\
\text { ACUM. }\end{array}$ & VALOR & PROPORCIÓN & $\begin{array}{l}\text { PROP. } \\
\text { ACUM. }\end{array}$ \\
\hline 1 & 2,13 & 0,53 & 0,53 & 1,65 & 0,41 & 0,41 \\
\hline 2 & 0,98 & 0,24 & 0,78 & 1,27 & 0,32 & 0,73 \\
\hline 3 & 0,59 & 0,15 & 0,93 & 0,98 & 0,24 & 0,98 \\
\hline 4 & 0,3 & 0,07 & 1 & 0,1 & 0,02 & 1 \\
\hline \multicolumn{7}{|c|}{ Correlaciones con las variables originales } \\
\hline Variables & CP 1 & CP 2 & CP 3 & CP 1 & CP 2 & CP 3 \\
\hline T med. $\left({ }^{\circ} \mathrm{C}\right)$ & 0,88 & $-0,22$ & 0,12 & 0,71 & 0,26 & $-0,64$ \\
\hline Ac/At & 0,72 & 0,45 & 0,49 & $-0,3$ & 0,9 & $-0,26$ \\
\hline Az Sup/H. Sol & $-0,65$ & 0,7 & 0,13 & 0,36 & 0,61 & 0,7 \\
\hline $\mathrm{Al} \mathrm{C}$ & $-0,66$ & $-0,49$ & 0,57 & 0,96 & $-0,14$ & 0,12 \\
\hline \multicolumn{7}{|c|}{ Correlación cofenética $=1.000$} \\
\hline
\end{tabular}

FUENTE ELABORACIÓN PROPIA

\section{Temperaturas minimas}

De acuerdo con análisis de la matriz de correlaciones, para el caso de las temperaturas mínimas fueron seleccionados los siguientes indicadores:

- Relación abierto central/ abierto total: indica la proporción de espacio abierto y su distribución espacial en la manzana (coincidente con los análisis precedentes).

- (SVF): caracteriza las condiciones radiativas para un punto de observación; expresa la relación entre área visible de cielo y la porción de bóveda celeste cubierta por los distintos componentes del medio. 
- Emisividad combinada: resulta del promedio de la emisividad de los materiales, ponderada por área horizontal en la manzana.

Los resultados mostraron que, de 30 días analizados, el comportamiento térmico de 24 días se explica entre un $92 \%$ y un $99 \%$ por el efecto de estos tres indicadores (tabla 9).

La variable objeto de estudio -relación abierto central/abierto total- explica las temperaturas mínimas de la manzana en primer lugar un $67 \%$ de las veces, con rangos de explicación del fenómeno del 36\% al 50\%; en segundo lugar, un 29\% de las veces con rangos de explicación del 28\% al 37\%; y en tercer lugar, el 4\% de las veces con rangos de $17 \%$.

Para el total de los días evaluados, el análisis por componentes principales ACP de las temperaturas mínimas indica que, en el $71 \%$ de los días, la manzana con una distribución dispersa del espacio abierto (caso 2) es más fresca respecto de la manzana con el espacio abierto concentrado en el centro (caso 1). Es decir, en la tabla de correlaciones con las variables originales (tabla 9), cuando la relación espacio abierto central respecto al espacio abierto total crece, la temperatura mínima aumenta.

Lo anterior implica que la hipótesis de que la distribución del espacio abierto de forma dispersa en la manzana posee beneficios para el enfriamiento, se verifica estadísticamente en el total de los días analizados.

TABLA 9 Resultados de ACP con relación abierto central/abierto total explicada en primer lugar para el día 04.02.15 y explicada en segundo lugar para el día 23.01.15

\begin{tabular}{|c|c|c|c|c|c|c|}
\hline \multicolumn{7}{|c|}{ AUTOVALORES } \\
\hline LAMBDA & VALOR & PROPORCIÓN & $\begin{array}{l}\text { PROP. } \\
\text { ACUM. }\end{array}$ & VALOR & PROPORCIÓN & $\begin{array}{l}\text { PROP. } \\
\text { ACUM. }\end{array}$ \\
\hline 1 & 1,71 & 0,43 & 0,43 & 1,77 & 0,44 & 0,44 \\
\hline 2 & 1,29 & 0,32 & 0,75 & 1,22 & 0,31 & 0,75 \\
\hline 3 & 0,96 & 0,24 & 0,99 & 0,91 & 0,23 & 0,98 \\
\hline 4 & 0,04 & 0,01 & 1 & 0,1 & 0,02 & 1 \\
\hline \multicolumn{7}{|c|}{ Correlaciones con las variables originales } \\
\hline Variables & CP 1 & CP 2 & CP 3 & СР 1 & СР 2 & CP 3 \\
\hline $\mathrm{T}$ min. $\left({ }^{\circ} \mathrm{C}\right)$ & 0,9 & 0,38 & $-0,17$ & 0,87 & 0,38 & 0,24 \\
\hline $\mathrm{Ac} / \mathrm{At}$ & 0,9 & $-0,39$ & $-0,16$ & $-0,28$ & 0,84 & 0,46 \\
\hline SVF & 0,13 & 0,91 & 0,39 & 0,43 & $-0,59$ & 0,68 \\
\hline $\mathrm{EmC}$ & 0,28 & $-0,41$ & 0,87 & $-0,86$ & $-0,18$ & 0,43 \\
\hline
\end{tabular}

FUENTE ELABORACIÓN PROPIA 


\section{Conclusiones}

Los resultados obtenidos muestran la importancia de revisar las normativas que determinan la distribución de las superficies abiertas en las manzanas atendiendo a su rol como espacio regulador de las condiciones microclimáticas del entorno construido mediato.

Se observó que la única diferencia importante entre los indicadores fue la concentración. Estos resultados indican que es más beneficiosa para su entorno una manzana con un esquema de espacio abierto disperso en verano que aquella con espacio abierto concentrado.

En relación con el problema de investigación, que se basa en establecer la forma más eficiente de distribución del espacio abierto en la manzana urbana, se demuestra que la manzana con distribución de espacio abierto disperso (caso 2) es más fresca que la manzana con espacio abierto concentrado en el centro (caso 1).

De acuerdo con el análisis de frecuencias por orientación, se observaron mayores temperaturas en el caso de la manzana con espacio abierto concentrado en el centro (caso 1). Estos resultados se desprenden de ambos conjuntos de análisis (58 días y 30 días representativos). La tendencia indicada se observó en alrededor de un $68 \%$ del total de los casos evaluados, acentuándose en los casos monitoreados con orientación oeste $(82 \%)$ y norte $(71 \%)$, siendo menos notoria en los casos con orientación sur $(53 \%)$.

En términos generales, se verificó mayor porcentaje de rangos de frecuencia positivos para las cuatro orientaciones de los canales viales, producto de la diferencia del caso 1 versus el caso 2, indicándose que el entorno de la manzana con distribución concentrada del espacio abierto (caso 1), obtuvo mayor calentamiento que la manzana con espacio abierto disperso (caso 2).

Respecto al cálculo de frecuencias (máximas, medias y mínimas), esta tendencia se mantiene en las tres categorías, siendo más clara en las máximas y en las mínimas (71\% y $78 \%$, respectivamente).

Para fortalecer este análisis se realizó un ACP orientado a valorar estadísticamente qué características de la manzana son responsables del comportamiento térmico de los canales viales que rodean a la misma. Se determinó un conjunto de indicadores descriptivos de distintos aspectos de las manzanas urbanas evaluadas: volumen, compacidad, altura media, relación sellado/abierto, relación abierto central/abierto total, azimut superficial, azimut solar en temperatura máxima, azimut superficial/azimut solar en temperatura máxima, horas de sol, azimut solar temperatura máxima/horas de sol, azimut superficial/horas de sol, factor de visión del cielo/horas de sol, factor de ocupación del suelo, factor de ocupación total, albedo combinado, factor de visión del cielo y emisividad combinada.

Los resultados muestran que entre el $71 \%$ y $100 \%$ de los días monitoreados, las temperaturas máximas, medias y mínimas de los canales viales que rodean una manzana pueden explicarse, entre un $86 \%$ y un $99 \%$, por la incidencia de tres indicadores: 
- Para el caso de las temperaturas máximas y medias, la combinación entre relación abierto central/abierto total, azimut superficial/horas de sol y albedo combinado.

- Para el caso de las mínimas, relación abierto central/abierto total, svf y emisividad combinada.

El único indicador explicativo que se repite en la totalidad de las situaciones evaluadas es la relación abierto central/abierto total de la manzana.

En todos los análisis, a medida que el espacio abierto de la manzana se concentra en el centro, las temperaturas microclimáticas urbanas se incrementan.

Este resultado pone en relevancia la influencia de las características de la distribución del espacio abierto de una manzana urbana en relación con las temperaturas de su entorno inmediato. De ello se desprende que es importante profundizar el estudio de este indicador y examinar cómo se lo debe regular a través de los códigos de edificación urbana para disminuir el impacto de la trama edilicia sobre el microclima de los canales viales adyacentes dentro de la ciudad, lo cual repercute significativamente sobre los consumos energéticos, la calidad del aire y el confort de los habitantes de la misma.

Como continuidad de este trabajo se podrían conseguir más diferenciaciones, aplicando distintas tecnologías y modificando las propiedades superficiales. Además, sería factible proponer un modelo en donde se puedan introducir estos indicadores para obtener resultados concretos.

Las conclusiones son válidas para la condición de verano. En un futuro se propone evaluar la condición de invierno, para tener una visión acabada de los efectos de las configuraciones de manzanas urbanas en el ambiente térmico de los espacios.

\section{Referencias bibliográficas}

Alchapar, N., Correa, E. \& Cantón, M. (2014). Envelopes according their capacity for mitigation the urban heat island in semiarid zones. Energy and Buildings, (69), 22-32. http://dx.doi.org/10.1016/j.enbuild.2013.10.012

Cantón, M. A. (2004). Centros libres de manzana en el Área Metropolitana de Mendoza. Análisis y diagnóstico de la condición del espacio abierto. Avances y Energías Renovables y Medio Ambiente, 8(1), 31-36. http://www.cricyt.edu.ar/asades/modulos/averma/ trabajos/2004/2004-t005-a006.pdf

Cantón, M. A., Fernández, J., Mesa, A. \& de Rosa, C. (2006). Energy assessment of the patiohouse evolution in the urban architecture of arid zones. Seminario internacional: Living in deserts: Is a sustainable urban design still possible in arid and hot regions? (pp. 55-68). Ghardaïa, Argelia, 9-12 diciembre de 2006.

Clark, J. R. \& Matheny, N. P. (1998). A model of urban forest sustainability: application to cities in the United States. Journal of Arboriculture, 24(2), 112-120. https:// urbanforestrysouth.org/resources/library/citations/a-model-of-urban-forestsustainability-application-to-cities-in-the-united-states 
Correa, E. N., Pattini, A., Córica, M. L., Fornés, M. \& Lesino, G. (2006). Software: Pixel de Cielo. Programa informático que calcula el factor de visión de cielo en un punto determinado de un área de estudio, a partir del procesamiento de una imagen digital hemisférica tomada con una lente ojo de pez. Número de registro CONICET: 07304 (2006-VIGENTE).

Correa, E., Martínez, C., Lesino, G., de Rosa, C. \& Cantón, A. (2006). Impact of urban parks on the climatic pattern of Mendoza's metropolitan area in Argentina. PLEA2006 - The $23^{\text {rd }}$ Conference on Passive and Low Energy Architecture. Génova, 6-8 de septiembre de 2006.

Correa E. N., De Rosa C. \& Lesino, G. (2008). Urban heat island effect on heating and cooling degree day's distribution in Mendoza's metropolitan area. Environmental costs. Proceedings of the EUROSUN 2008, 1st International Conference on solar heating, cooling and buildings (pp. 951-958). Lisboa, Portugal, Sociedade Portuguesa de Energia Solar (sPEs), 7-10 de octubre de 2008.

Duffie, J. A. \& Beckman, W. A. (1991). Solar engineering of thermal processes (2 ${ }^{\mathrm{da}}$ ed.). Nueva York: Wiley Interscience.

Hernández, A. L. (2003). geosol: una herramienta computacional para el cálculo de coordenadas solares y la estimación de irradiación solar horaria. Avances en Energías Renovables y Medio Ambiente, 7(2), 11.19-11.24. http://www.cricyt.edu.ar/asades/ modulos/averma/trabajos/2003/2003-t011-a004.pdf

Oke, T. R. (2006). Initial guidance to obtain representative meteorological observations at urban sites. Instruments and Observing Methods (Iом) Report No. 81 [wмo/TD-No. 1250]. Génova: World Meteorological Organization (wMo). https://library.wmo.int/pmb_ ged/wmo-td_1250.pdf

Pantavou, K., Theoharatos, G., Mavrakis, A. \& Santamouris, M., 2011. Evaluating thermal comfort conditions and health responses during an extremely hot summer in Athens. Build. Environ. 46(2), 339-344. http://dx.doi.org/10.1016/ j.buildenv.2010.07.026.

Rosenfeld, A., Akbari, H., Romm, J. \& Pomerantz, M. (1998). Cool communities: strategies for heat island mitigation and smog reduction. Energy and Buildings, 28(1), 51-62. https://doi.org/10.1016/S0378-7788(97)00063-7

Ruiz, A. \& Correa, E. (2015a). Adaptive model for outdoor thermal comfort assessment in an Oasis city of arid climate. Building and Environment, (85), 40-51. https://doi. org/10.1016/j.buildenv.2014.11.018

Ruiz A. \& Correa E. (2015b). Suitability of different comfort indices for the prediction of thermal conditions in tree-covered outdoor spaces in arid cities. Theoretical and Applied Climatology, 122(1-2), 69-83. https://doi.org/10.1007/s00704-014-1279-8

Santamouris, M. (ed.). (2001). Energy and climate in the urban built environment. Londres: James \& James Science Publishers.

Stathopoulou, E., Mihalakakou, G., Santamouris, M. \& Bagiorgas, H. (2008). On the impact of temperature on tropospheric ozone concentration levels in urban environments. Journal of Earth System Science, 117(3), 227-236. http://dx.doi.org/10.1007/s12040008-0027-9 